\title{
Operation of Passive Fluid Self-Balancing Device at Resonance Transition Regime
}

\author{
Vilen ROYZMAN*, Ilona DRACH**, Viktor TKACHUK***, Kęstutis PILKAUSKAS****, \\ Ginas ČIŽAUSKAS*****, Anatolijus ŠULGINAS****** \\ *Khmelnitsky National University, Instutytska 11, 29016 Khmelnitsky, Ukraine, E-mail: iftomm@ukr.net \\ **Khmelnitsky National University, Instutytska 11, 29016 Khmelnitsky, Ukraine, E-mail: cogitare410@gmail.com \\ ***Khmelnitsky National University, Instutytska 11, 29016 Khmelnitsky, Ukraine, E-mail: tkachukv.p@gmail.com \\ ****Kaunas University of Technology, Studentu 56, LT-51424 Kaunas, Lithuania, E-mail: kestutis.pilkauskas@ktu.lt \\ ******Kaunas University of Technology, Studentu 56, LT-51424 Kaunas, Lithuania, E-mail: ginas.cizauskas@ktu.lt \\ *******Kaunas University of Technology, Studentu 56, LT-51424 Kaunas, Lithuania, E-mail: anatoljus.sulginas@ktu.lt \\ crossref http://dx.doi.org/10.5755/j01.mech.24.6.22469
}

\section{Introduction}

For the class of machines with variable rotor imbalance (centrifuges, separators, etc.), traditional balancing methods are ineffective. The only balancing method is automatic balancing due to free movement of correction counterweights. There a number of patents for automatic selfbalancing devices (SBD), but majority of them have no potential to be implemented practically. The researches state that this type of balancing is possible only at above resonance rotational frequencies. However, there is no theoretical proof for this condition. Meanwhile results of experimental research of the authors reveal that this kind of balancing is possible under and below resonance frequencies as well. The research of the operation of passive fluid selfbalancing device when the rotor is passing resonance is presented.

\section{Research of the operation of passive fluid self-balanc- ing device when rotor is passing resonance}

Reduction of oscillation level in machines is possible by using both artificial and natural damping. While the oscillation excitation source remains unaffected, this mainly results in the decrease of oscillation level of frames and foundations. Traditional methods are ineffective for machines with variable rotor imbalance and for balancing machine rotors without a halt for maintenance procedures. The only possible the most perspective and reliable method of vibration reduction in such machines is automatic balancing by means of free movement of correcting masses [1].

The phenomenon - self-balancing of a rotor with the help of fluid media moving in partly filled by it cavity chambers that function as passive regulators which need no energy supply and control system for the movement of correcting masses is proposed.

The history of fluid automatic balancing begins in 1916, when French engineer Leblanc proposed fluid SBD (self-balancing device) for balancing the extractor of washing machine during its operation. Later the Thearle's SBD, Duncan's SBD, and well-known Leblanc's SBD improvements type were proposed. For the first time E. L. Thearle made an attempt to find theoretical background for operating principle of circular, pendulous and spherical Leblanc's SBD in the series of his articles [2]. He proposed planar model of the rotor and SBD. Within its limits the rotor has one critical rotation frequency and when it is exceeded the rotor starts rotating with the light side outwards and the effect of rotor self-centering appears. This law is taken as the background of operation principle of all passive SBDs. The results of E.L. Thearle research were used as the basis by other researches: J. Larri, V. I. Susanin, L. N. Shatalov, B. N. Grushin, V. I. Kravchenko, V. P. Nesterenko, M. W. M. Cunico, E. N. Pashkov, Kim Hyun Min, Seiichsrou Suzuk, Tatsumi Hisao AS. Nevertheless, the obtained without taking into consideration specific features of correcting weight (CW), resistance forces, theoretically ungrounded and experimentally untested efficiency range of this self-balancing devices above critical (or above resonance) rotor rotation zone is not studied until now [3-5].

In 1994 multichamber fluid self-balancing device for washing machine with horizontal arrangement of the barrel, designed by scientists of Whirlpool Corporation was patented. However, there is no information about practical realization of devices for automatic balancing of rotors mentioned above [6]. The operation of passive fluid self-balancing device when the rotor is passing the resonance is being studied.

\section{Theoretical part}

The research of the operation of passive fluid selfbalancing devices for rotors with variable imbalance was carried out in several phases.

In the first phase mathematical modeling of stationary motion in rigid supports of a rigid rotor with a cylindrical chamber partially filled with fluid was carried out. The cases of unlimited and limited chamber eccentrically and symmetrically located on the top of the shaft were analysed. Fluid behavior in self-balancer is analysed without taking into account external damping. It is specified that the fluid is symmetric about the axis of rotation of the system. Its main mass is concentrated on the part of eccentricity (with eccentric arrangement of the chamber). Analysis of the constructed models indicates that it is impossible to balance automatically rigid rotor in rigid supports with a fluid. A detailed description of the results of the first phase of research is given in $[7,8]$.

At the next Phase the mechanical system of a solid body with a cylindrical chamber partly filled with a fluid is investigated, and mathematical model of its stationary motion is derived [9]. 
The mathematical model of fluid behavior in SBD, was derived using the following properties of fluids:

- the fluid volume can arbitrarily change its shape under the influence of any small forces.

- the viscosity forces are taken into account only when considering relatively fast movements, when the rate of changes in the fluid is relatively high.

The background of the mathematical model [10] is the principle of least action in the form of Hamilton-Ostrogradsky:

$$
\delta W P=-0.5 \frac{k_{0}^{2}}{I_{0}^{2}} \delta I=0,
$$

where: $W=0.5 \frac{k_{0}^{2}}{I}+P$ is the change of potential energy of the Solid-Fluid System; $P$ is the potential energy due to gravity and centrifugal force; $k_{0}$ is momentum in case of uniform rotation of the entire system as a single solid about fixed line with angular velocity $\omega ; I_{0}$ is the value of $I$ (mass moment of inertia of the system relative to the stationary axis) for steady motion.

Eq. (1) with $\omega I=k_{0}$ can be reduced to equations in the generalized coordinates of a solid $q_{j}(j=1, \ldots, n-1)$ in steady motion:

$$
\frac{\partial W}{\partial q_{j}}=-\frac{1}{2} \omega^{2} \frac{\partial I}{\partial q_{j}}+\frac{\partial P}{\partial q_{j}}=0(j=1, \ldots, n-1),
$$

and to equations for pressure in a fluid that allow obtaining an equation of its free surface in this motion (the motion of a fluid is described relative to moving coordinate system attached to the body):

$$
\rho U+\frac{1}{2} \rho \omega^{2}\left(x^{2}+y^{2}\right)-\alpha\left(\frac{1}{R_{1}}+\frac{1}{R_{2}}\right)=\text { const },
$$

where: $U$ is function of power of forces acting on fluid particles; $\rho$ is density of fluid, $R_{i}$ is main radii of curvature of free surface of fluid; $\alpha$ is the coefficient of surface tension.

$k_{0}$ can be considered as a parameter and apply to the mechanical system under consideration of the results of the general theory of "equilibrium" of material systems with potential energy, which depends on the parameter.

For fixed parameter value of $k_{0}$ Eqs. (2) and (3) determine the coordinates of the solid and the shape of the free surface of the fluid in stationary motion.

To consider mechanical system without surface tension of fluid, potential energy of the system and its inertia moment relative to axis $x_{3}^{\prime \prime}$, are defined by the formulas:

$$
\begin{aligned}
& P=M g\left(x_{c 1} \gamma_{1}+x_{c 2} \gamma_{2}+x_{c 3} \gamma_{3}\right), \\
& I=A \gamma_{1}^{2}+B \gamma_{2}^{2}+C \gamma_{3}^{2}-2 D \gamma_{2} \gamma_{3}-2 E \gamma_{1} \gamma_{3}-2 F \gamma_{1} \gamma_{2},
\end{aligned}
$$

where: $A, B, C$ are axial inertia moments, $D, E, F$ are centripetal inertia moments of system; $M$ is system mass; $x_{c 1}$, $x_{c 2}, x_{c 3}$ are coordinates of gravity center of system; $\gamma_{i}$ is projections of unit vector $i_{3}^{\prime \prime}$ of immobile axis $x_{3}^{\prime \prime}$ to mobile axis are related by the equation:

$$
\gamma_{1}^{2}+\gamma_{2}^{2}+\gamma_{3}^{2}=1
$$

Given this equality, explicitly written equations of stationary motions are:

$$
\begin{aligned}
& \frac{\partial W}{\partial \gamma_{1}}=-\frac{1}{2} \omega^{2} \frac{\partial\left(A \gamma_{1}^{2}+B \gamma_{2}^{2}+C\left(1-\gamma_{1}^{2}-\gamma_{2}^{2}\right)-2 D \gamma_{2} \sqrt{1-\gamma_{1}^{2}-\gamma_{2}^{2}}-2 E \gamma_{1} \sqrt{1-\gamma_{1}^{2}-\gamma_{2}^{2}}-2 F \gamma_{1} \gamma_{2}\right)}{\partial \gamma_{1}}+ \\
& +\frac{\partial\left(M g\left(x_{c} \gamma_{1}+y_{c} \gamma_{2}+z_{c} \gamma_{3}\right)\right)}{\partial \gamma_{1}}=0, \\
& \frac{\partial W}{\partial \gamma_{2}}=-\frac{1}{2} \omega^{2} \frac{\partial\left(A \gamma_{1}^{2}+B \gamma_{2}^{2}+C\left(1-\gamma_{1}^{2}-\gamma_{2}^{2}\right)-2 D \gamma_{2} \sqrt{1-\gamma_{1}^{2}-\gamma_{2}^{2}}-2 E \gamma_{1} \sqrt{1-\gamma_{1}^{2}-\gamma_{2}^{2}}-2 F \gamma_{1} \gamma_{2}\right)}{\partial \gamma_{2}}+ \\
& +\frac{\partial\left(M g\left(x_{c} \gamma_{1}+y_{c} \gamma_{2}+z_{c} \gamma_{3}\right)\right)}{\partial \gamma_{2}}=0 .
\end{aligned}
$$

Solution of these equations when:

$$
\gamma_{1}=\gamma_{2}=0, \gamma_{3}=0
$$

at any value $\omega(\omega \neq 0)$, if mass center of the system is located on the axis of its rotation, which is at the same time the main central axis of inertia, corresponds to the condition of selfbalancing.

The next research phase is mathematical modeling of fluid transfer process in the ABP cell under the influence of total imbalance change and deflection of the system. Operation of the simplest rotary system, with SBD, in the form of $R$ radius cylindrical cavity and fluid height $h$, for a vertical flexible rotor on two rigid supports, is analysed when rotating at different angular velocities values $(\omega=0$, $\left.\omega<<\omega_{\text {res }}, \omega<\omega_{\text {res }}, \omega \approx \omega_{\text {res }}, \omega>\omega_{\text {res }}\right)$. If we take into account damping forr a rotor with fluid SBD and, the location of deflection plane $I-I$ and imbalance plane $\bar{D}_{c}$ for the rotor system - fluid at a point in time will be as shown in Fig. 1. For a fluid volume, in general case only the positions of the system of points that are not tightly bound together are valid.

Let us introduce the denotation. Total imbalance of the rotor system $\bar{D}_{c}$ consists of shaft imbalance $\bar{D}_{0}$ and fluid imbalance $\bar{D}$. Let $O$ be the point of intersection of the axis of the curved shaft with the plane of the cavity (Fig. 1); $O-O_{1}$ is bearing line; $C_{0}$ is center of masses of the shaft, $C$ is center of masses of the fluid, $C_{c}$ is center of masses of the 
system shaft-fluid; $M$ is weight of shaft, $m$ is mass of the fluid; $\overline{O C}=\bar{e}=\frac{\bar{D}}{(m+M)}$ is "oriented" eccentricity of the mass center of the fluid in the system SBD is fluid; vector $\overline{O C}_{0}=\bar{e}_{0}=\frac{\bar{D}_{0}}{(m+M)}$ is "oriented" eccentricity of the rotor mass $M ; \overline{O C}_{c}=\bar{e}_{c}=\frac{\bar{D}_{c}}{(m+M)}$ is "oriented" total eccentricity of the rotor with a fluid.

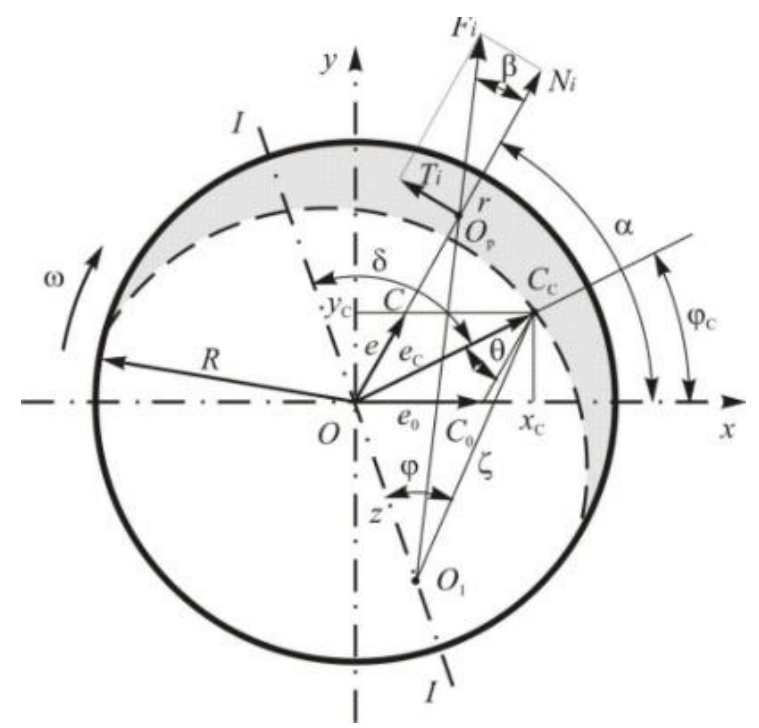

Fig. 1 Forces in the SBD in case of resistance

To study the position of the fluid relative to the shaft imbalance and the balancing efficiency, the geometric model of the system is used (Fig. 1) taking into account external damping.

When angular velocity $\omega<\omega_{\text {res }}$ external resistance (forces of friction in bearings, forces of friction of a cylinder moving in air, etc.) causes a backlog of a deflection plane $(I-I)$ from the plane of total imbalance $\left(O C_{\mathrm{c}}\right)$ at the angle $\delta$ (Fig. 1).

Tangential component $\bar{T}$ of centrifugal forces of inertia $\bar{F}$ (Fig. 1) and the specific properties of the fluid facilitate the transfer of fluid in the SBD chamber to the position that corresponds to reduction in overall imbalance of the system, even at subcritical speeds.

The fluid tends to occupy the most distant position from the axis of rotation, i.e. in the trough. This leads to the change in total imbalance of the system in terms of direction and value. Since for this angular velocity deflection angle from total imbalance $\delta$ is constant, with the change in direction of the total imbalance, the position of deflection changes with respect to the initial imbalance $\bar{D}_{0}$ (i.e., the angle of this lag with respect to the initial imbalance increases). The fluid following the deflection again further the total imbalance, increasing angle $\alpha$ and reducing magnitude of the total imbalance. This leads to decrease in deflection and to the change of its position relative to $\bar{D}_{0}$ (lag angle increase). The process proceeds till the lag angle value of $180^{\circ}$ and minimum value deflection $\alpha$ is reached. In the position $\alpha$ total imbalance of the rotor is $\bar{D}_{c}=(m+M) \bar{e}_{c}$. From Fig. 1:

$$
\begin{aligned}
& e_{c}=\sqrt{x_{c}{ }^{2}+y_{c}{ }^{2}}=\sqrt{(e(\cos \alpha+k))^{2}+(e \sin \alpha)^{2}}= \\
& =e \sqrt{1+2 k \cos \alpha+k^{2}} .
\end{aligned}
$$

where: $k=\frac{D_{0}}{D}$ is the ratio of rotor and fluid imbalances, $e=\frac{m}{(m+M)} \frac{r^{2}}{R^{2}-r^{2}} f$ is the radius of free surface of the fluid (free surface of the fluid is assumed as circular with the center at point $\left.O_{1}\right), f=O_{1} O$ is deflection $f=e_{c} \cos \delta\left[\frac{\cos \phi \omega_{r e s}^{2}}{\omega_{k p}^{2}-\omega^{2}}-1\right]$

The condition of fluid equilibrium(Fig. 1) eglecting the forces of tension is non existance of tangential component $\operatorname{tg} \beta=0$. Analytically:

$$
f\left[\operatorname{tg} \alpha \cos \left(\delta+\phi_{c}\right)-\sin \left(\delta+\phi_{c}\right)\right]=0 .
$$

or:

$$
\begin{aligned}
& f=0 ; \\
& \operatorname{tg} \alpha_{0} \cos \left(\delta+\phi_{0 c}\right)-\sin \left(\delta+\phi_{0 c}\right)=0 .
\end{aligned}
$$

Eq. (5) corresponds to the case with no deflection, which contradicts the condition of the problem for an elastic-deformable rotor. From Eq. (6) follows the equilibrium of fluid:

$$
\operatorname{tg} \alpha_{0}=\operatorname{tg}\left(\delta+\phi_{0 c}\right) \text {. }
$$

Given the value $e, e / e_{0}, e_{c}$ and $\theta=\alpha-\varphi_{c}$,

$$
\text { in } \alpha_{0}=\frac{1}{k} \sin \delta \sqrt{1+2 k \cos \alpha_{0}+k^{2}} \text {. }
$$

From Eq. (6) in case of no external resistance in the system ( $\delta=0, k \neq 0$ ) the equilibrium position angle of the fluid coincides with the position of the rotor imbalance ( $\alpha=0$ ), what allows concluding that in the system with no external damping the fluid increases the imbalance.

After algebraic transformations of expression (7) with $\delta \neq 0, k \neq 0$ angle $\alpha_{0}$, which corresponds to the position of the fluid equilibrium ( $\beta=0)$ :

$$
\cos \alpha_{0}=\frac{-\sin ^{2} \delta \pm \sqrt{\sin ^{4} \delta-\left(1+k^{2}\right) \sin ^{2} \delta+k^{2}}}{k},
$$

where:

$$
\begin{aligned}
& \alpha_{0}=\pi- \\
& -\arccos \left(\sin ^{2} \delta+\sqrt{\left(\sin ^{2} \delta-1\right)\left(\sin ^{2} \delta-k^{2}\right)}\right) .
\end{aligned}
$$

Analysis of the expression (8), allows stating:

- $\quad$ arcos argument is positive therefore even at subcritical frequencies of rotor rotation at $\delta \in\left(0, \frac{\pi}{2}\right)$, $\alpha_{0} \in\left(\frac{\pi}{2}, \pi\right)$ 
- the efficiency of balancing is characterized by the ratio of deviations from the axis of rotation of the mass center of without and with fluid $\lambda_{0}$ :

$$
\lambda_{0}=\frac{e_{o}}{e_{c}}=\frac{k}{\sqrt{1+2 k \cos \alpha_{0}+k^{2}}} ;
$$

- in case of $1+2 k \cos \alpha_{0}+k^{2}=0$ there will be a full equilibrium, the only solution will be $k=1$ when $\cos \alpha_{0}=-1$, i.e. $\alpha_{0}=\pi$. Consequently, the greatest balancing effect is achieved when imbalance of the balancing fluid will be close to the initial value of the rotor imbalance $(k \approx 1)$.

The fluid, not functioning as balancing media, is distributed in concentric circles, without affecting the total imbalance of the system.

\section{Experimental research}

\subsection{Experimental model of automatic balancing device}

The experimental model of SBD is the ring with external diameter of $\varnothing 400 \mathrm{~mm}$, two concentric partitions with diameters of $\varnothing 300$ and $\varnothing 200 \mathrm{~mm}$ which form three concentric chambers for arrangement of working bodies. General appearance of it is shown in Fig. 2.

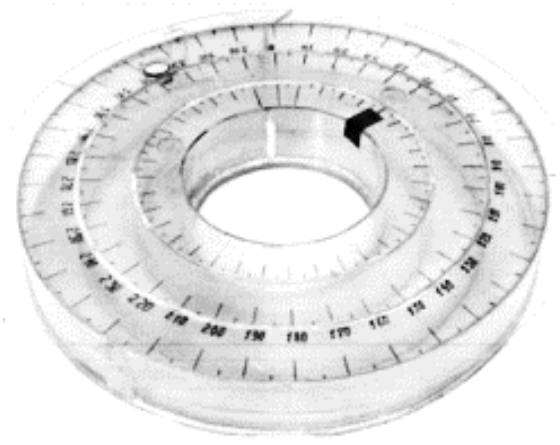

Fig. 2 General appearance of the SBD experimental model

All three chambers are isolated from each other what allows using simultaneously both fluid and rigid working bodies in the SBD. The water of different colors in each chamber was used as fluid working media for of the SBD. Metal and plastic balls with diameters of 6, 8, 12 and $16 \mathrm{~mm}$ were used as rigid working bodies. Metal and plastic balls with diameters of $1,1.5,2,4 \mathrm{~mm}$ were used as dry substances. The scale on the ring edge serves for determination of angular position of working bodies in SBD during the experiment. The position of imbalanced mass (imbalance) is fixed on this scale with the help of a thin color strip, of size $10 \times 20 \mathrm{~mm}$ with adhesive layer on one of its surfaces. The bottom of the SBD is matt surface in order to increase the contrast of visual observation of the working bodies' position. The rods fixing experimental model are attached to the external side surface of SBD at the angle of $120^{\circ}$.

\subsection{Structure of the test bench}

The research installation is a rigid cantilever vertical rotor, resiliently hung on the frame.

Rotor axis through self-aligned bearings is connected with a rigid platform which is joined to the frame of the installation by means of four resiliently damper brackets (typical vibration frequency of all the system on the brackets is equal to 2-2.5 Hz.). The SBD experimental model is set on the free end of the hollow cylinder (further - a drum). It is placed inside the drum and fixed to its external surface. For a high-velocity video recording the inner surface of the drum was illuminated by incandescent lamp of $200 \mathrm{~W}$, installed in the inner cavity of the rotor. The inner surface of the drum was made matt $r$.

4.3. Measuring, registering equipment and preparation of installation

Block scheme of the test bench is presented in Fig. 3.

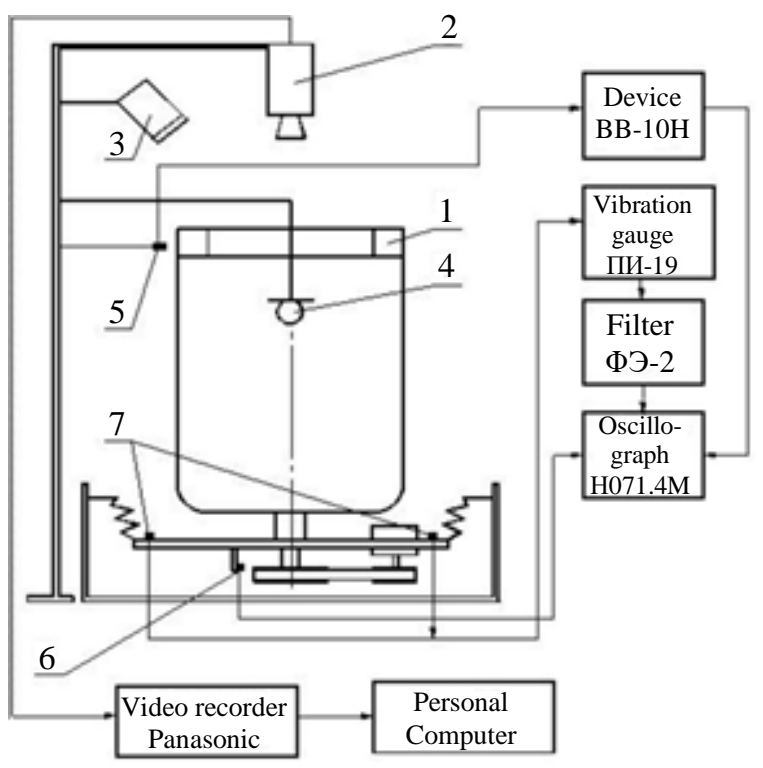

Fig. 3 Bloc-scheme of the test bench: $1-$ the SBD experimental model, 2 - video camera 3 - stroboscope, 4 incandescent lamp, 5 - induction data unit, 6 - revolutions data unit, 7 - accelerometers

Angular velocity of the rotor rotation was measured by noncontact method on the basis of Hall's data unit.

Accelerometers were installed on the rotor platform. Signal from the accelerometers was transferred to vibration gauges ПИ-19 where it was integrated twice in order to obtain displacement amplitudes of the system platformrotor.

Amplitudes of the rotor vibrations were measured by means of the elaborated optical electronic device.

Measurements were carried out as follows. The mass of artificial imbalance was increased with the increment of $5 \mathrm{~g}$ and vibration level of the rotor was measured.

The imbalance was considered as Critical maximum meaning of mass at which vibrations amplitude reached $35 \mathrm{~mm}$ (higher values cause impacts of moveable elements with the frame) or when the electric motor is not able to increase rotational frequency during transition through system resonance and the rotor «is suspended» at the critical rotation frequency.

As Criterion of optimum volume of the fluid or of quantity of working bodies in SBD was considered the visibility of the process of automatic balancing at all rotation frequencies of the rotor, i.e., when the position of working 
bodies or fluid in relation to the place of arrangement of artificial imbalance was clearly visual in the light of stroboscope and on the videotape. The volume determined by experiments was $0.45,0.3$ and $0.25 l$ for external, medium and internal chambers of the SBD correspondently.

\subsection{Video recording of working bodies movement in SBD} in stationary modes of the rotor rotation

Fig. 4 shows the most typical and representative computer slides of the video recording of the fluid position in SBD at under-resonance (Fig. 4, a; $\omega=8 \mathrm{c}^{-1}$ ), resonance (Fig. 4, b; $\omega=12.5 \mathrm{c}^{-1}$ ) and over-resonance (Fig. 4, c; $\omega=$ $\left.=90 \mathrm{c}^{-1}\right)$ frequencies of the rotor rotation with SBD.

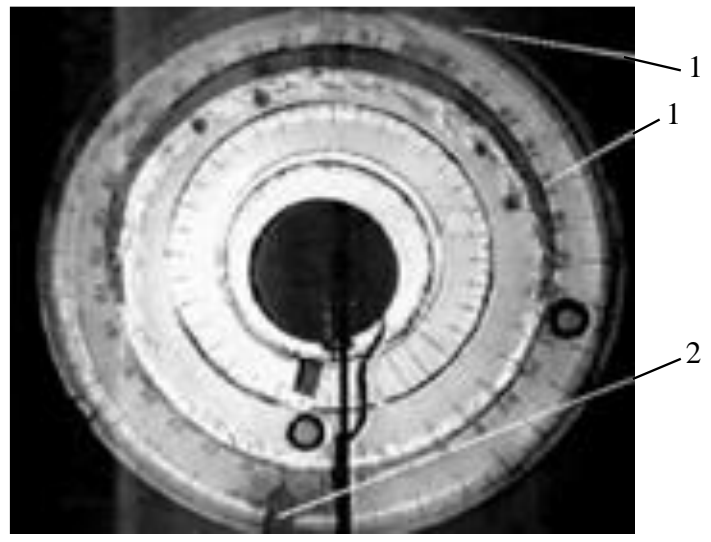

a

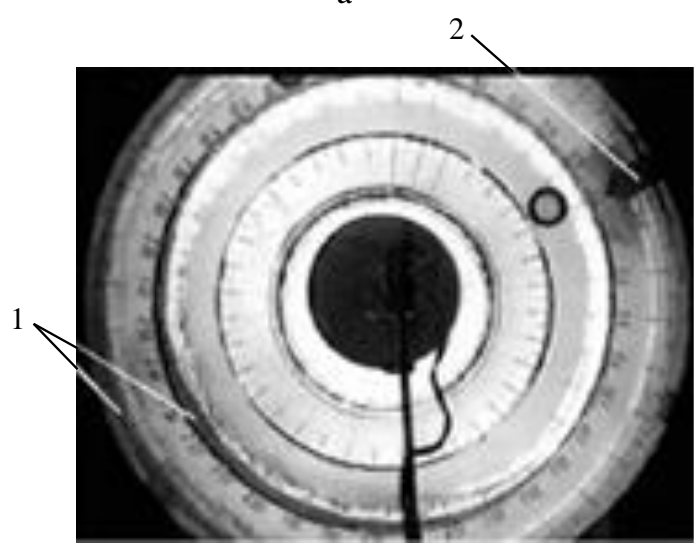

b

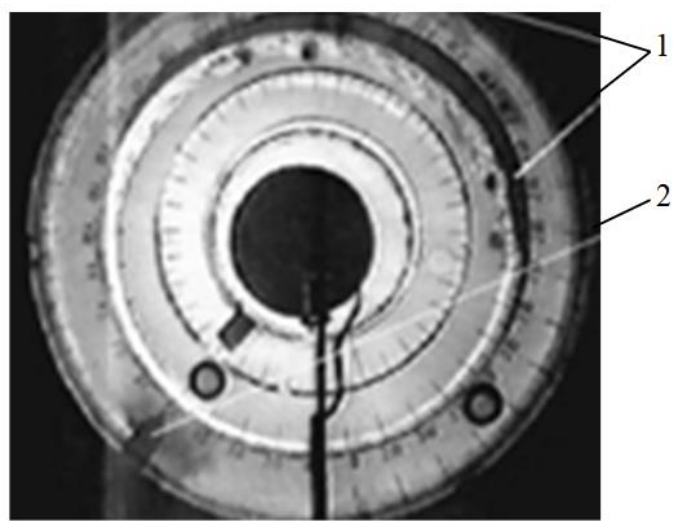

Fig. 4 Video images: $\mathrm{a}$ - before resonance, $\mathrm{b}$ - at resonance, $\mathrm{c}$ - beyond resonance. 1 - Fluid; 2 - Disbalance label
Analysis of these and other slides shows that the fluid compensates imbalance of the rotor at under-resonance, resonance and over-resonance frequencies of its rotation.

\section{Conclusions}

The results of experimental and theoretical research of fluid behavior in passive self-balancing devices, which are installed in rotors when passing resonance, are presented in this article.

The Received relations showed that automatic balancing by fluid is effective for elastically deformed rotors or (and) rotor on elastic supports, where the difference in phases between the direction of centrifugal force and flexure (or movement) of the rotor exists.

The obtained experimental results revealed that this difference in phases occurs when the rotor reaches resonance frequency of rotation and increases up to $180^{\circ}$ when passing the resonance. In this case the tangential force occurs, under the influence of which the fluid moves to the side of flexure, opposite to imbalance, and further equilibration of the rotor already at resonance rotation frequency occurs.

The influence of gravity under conditions of horizontal arrangement of SBD rotation axis, decreases tangential component, which moves fluid into the position opposite to imbalance and leads to decreasing of balancing accuracy in comparison with vertical rotation axis.

\section{References}

1. Gusarov, A. A. 2002. Autobalancing devices of direct action. - Moscow: Nauka, 119 p. (in Russian).

2. Thearle, E. L. 1950. Automatic dynamic balancers (Part 1. LeBlanc balancer), Machine Design 22: 119-124.

3. Thearle, E. L. 1950. Automatic dynamic balancers (Part 1. LeBlanc balancer), Machine Design 22: 119-124.

4. Kim Hyun Min, A. 1995. Study on the dynamic characteristics of the suspension of washing machine, The Second DADS Korean User's Conference '95, 49-60 p.

5. Marlon Wesley Machado Cunico. 2015. Characterization and modelling of LeBlanc hydrodynamic stabilizer: a novel approach for steady and transient state models, Modelling and Simulation in Engineering, 2015: 729582, $11 \mathrm{p}$. http://dx.doi.org/10.1155/2015/729582.

6. Pashkov, E. N.; Martyushev, N. V.; Ponomarev, A. V. 2014. Efficiency of balancing by liquid-type automatic balancing devices, Advanced Materials Research 1040: 858-863.

https://doi.org/10.4028/www.scientific.net/AMR.1040.858.

7. Pat.6782722 USA. Drum washing machine. Yokoi e.a. Sanyo Electric Co., Ltd.; 31.08. 2004.

8. Royzman, V; Drach, I. 2005. Improving theory for automatic balancing of rotating rotors with liquid self-balancers, Mechanika 4 (54). 38-44.

9. Royzman, V.; Bubulis, A.; Drach, I. 2009. System analysis of automatic balancing (self-balancing) machine rotors with liquid working bodies, Solid State Phenomena 147-149: 374-379. https://doi.org/10.4028/www.scientific.net/SSP.147149.374 . 
10. Royzman, V.; Drach, I.; Bubulis, A. 2016. Movement of working fluid in the field of centrifugal forces and forces of weight, In: Proceedings of the 21st International Scientific Conference "Mechanika 2016", 222$224 \mathrm{p}$.

11. Dimentberg, F. M.; Shatalov, K. T., Gusarov, A. A. 1964. Oscillations of machines. Moscow: Mashinostroenie, 256-291 p. (in Russian).
V. Royzman, I. Drach, V. Tkachuk, K. Pilkauskas,

G. Čizauskas, A. Šulginas

OPERATION OF PASSIVE FLUID SELF-BALANCING DEVICE AT RESONANCE TRANSITION REGIME

S u m m a r y

The main challenge faced at developing rotary machines with variable imbalance is the task of their vibroactivity reduction. An effective method of balancing rotor machines at operation, i.e. without stopping is passive automatic balancing due to free movement of fluid. In the current research the of phenomenon self-balancing of a rotor with the help of fluid media moving in partly filled by it cavity chambers, with that function as passive regulators which need no energy supply and control system is analyzed. Performance of passive fluid self-balancing device when its rotor is passing resonance is presented. The results of theoretical research are verified by experiments.

Keywords: rotor, resonance, vibration, self-balancing, selfbalancing units, fluid self-balancing device (SBD).

Received December 06, 2018 Accepted December 12, 2018 